\title{
G-Lets: A New Signal Processing Algorithm
}

\author{
B. Rajathilagam \\ Assistant Professor \\ Department of Information \\ Technology \\ Amrita Vishwa \\ Vidyapeetham, Coimbatore, \\ India
}

\author{
Murali Rangarajan \\ Associate Professor \\ Department of Chemical \\ Engineering and Materials \\ Science \\ Amrita Vishwa \\ Vidyapeetham, Coimbatore, \\ India
}

\author{
K.P Soman \\ Professor \\ Center for Excellence in \\ Computational Engineering \\ and Networking \\ Amrita Vishwa \\ Vidyapeetham, Coimbatore, \\ India
}

\begin{abstract}
Different signal processing transforms provide us with unique decomposition capabilities. Instead of using specific transformation for every type of signal, we propose in this paper a novel way of signal processing using a group of transformations within the limits of Group theory. For different types of signal different transformation combinations can be chosen. It is found that it is possible to process a signal at multiresolution and extend it to perform edge detection, denoising, face recognition, etc by filtering the local features. For a finite signal there should be a natural existence of basis in it's vector space. Without any approximation using Group theory it is seen that one can get close to this finite basis from different viewpoints. Dihedral groups have been demonstrated for this purpose.
\end{abstract}

\section{General Terms}

signal processing, algorithm, group theory.

\section{Keywords}

signal processing, transformation groups, group theory, multiresolution analysis.

\section{INTRODUCTION}

A digital signal is finite-dimensional. Therefore, within the approximation of digitization, the signal exists in a vector space of finite basis. If it is possible to obtain this basis of the signal space, a signal processing algorithm may be developed wherein the signal is projected onto the basis of the signal space to obtain its individual pieces. Such an algorithm would provide a general framework for extracting various features of interest from the signal without loss of information, within the digitization approximation. This is the inspiration behind the development of G-lets. Group theory [2][3][4][5][6] provides the crucial piece of the puzzle, namely the dimension of the vector space where the signal exists. Group theory also shows the way for choosing any suitable transformation or set of transformations required for a particular application customized to capture specific characteristics.

Fourier transform was first developed for continuous signals and then approximated for digital signals. In Wavelets, choosing a mother wavelet as an approximation of the signal, it's translates and dilates are the actual basis functions. Since translations cannot be finite, it is also an approximation. In Glets, we provide the most natural signal decomposition algorithm for a digitized signal without any artificially introduced approximations using general transformation groups.

\section{BACKGROUND}

Fourier series generate a representation for a signal in terms of sine and cosine functions, giving an infinite set of basis vectors for the signal. Here time and frequency information cannot be localized on specific parts of the signal. Generalized Fourier basis is formed by creating representation matrices of the transformations and calculating their trace, which are called the characters of the transform. Wavelets [8][9] create orthogonal subspaces using the transformations dilation and translation to capture different frequencies of the signal. Still, due to the edge effect produced by breaking frequencies into smaller and smaller pieces, in applications like face recognition they are unable to testify a good match.

Ridgelet transform [10][11] focuses on detecting lines in an image using Radon transform [12]. Curves are specially recognized in a signal by curvelets [13], which is a combination of wavelets and ridgelets. These two transforms are specialized forms of wavelets. There are also other specialized forms of wavelets like the contourlets [14], wedgelets [15], and grouplets [16]. In grouplets, the wavelet coefficients are grouped together based on the direction of their flow using multiscale association fields. Representation theory using rotations and reflections have been used by Lenz [17][18] for edge detection in images but they do not generalize the use of transformation groups to generate an orthogonal basis. In a recent work by Lenz, octahedral transformation group has been chosen to be suitable for a specific application, i.e., the 3-D image environment, and representations have been used to describe this environment by evaluating their correlation coefficients. Vale [19] shows how an orthonormal set of polynomials can be generated by the orbit of a vector using representation theory [20][21]. In this work, we focus on using all the transforms of a transformation group using transformations like rotations, reflections, dilations, translations and more through their irreducible representations producing a series of block diagonal matrices portraying the features of the signal from low to high frequencies progressively. Dihedral groups [22][23] have been chosen for the illustrations.

\section{GROUP THEORETICAL APPROACH}

A group is an ordered pair $\left(\mathrm{G},{ }^{*}\right)$, where $\mathrm{G}$ is a set and * is a binary operation on $\mathrm{G}$ satisfying the following axioms:

Closure : For all a,b $\varepsilon \mathrm{G}$, the product $\mathrm{a} * \mathrm{~b}$ is also in $\mathrm{G}$. Associativity: For all a,b,c $\varepsilon$ G, $(\mathrm{a} * \mathrm{~b}) * \mathrm{c}=\mathrm{a} *(\mathrm{~b} * \mathrm{c})$.

Identity: There exist an element $\mathrm{c} \varepsilon \mathrm{G}$, called the identity of $\mathrm{G}$, such that for all a $\varepsilon \mathrm{G}, \mathrm{a}^{*} \mathrm{c}=\mathrm{c} * \mathrm{a}=\mathrm{a}$. 
Inverse: For every element a $\varepsilon \mathrm{G}$, there is an element $\mathrm{a}^{-1}$, called the inverse of $a$, such that $a^{*} a^{-1}=a^{-1} * a=e$.

If the elements of the group are linear transformations, then it is called a transformation group [24]. A transformation group is a set $\mathrm{G}$ of transformations of a certain set which has the following two properties:

1. If two transformations $f$ and $g$ belong to $G$ then their composition $\mathrm{f}$ o $\mathrm{g}$ also belong to $\mathrm{G}$.

2. Together with every transformation $f$ the set $G$ also contains the inverse transformation $\mathrm{f}-1$.

Any two transformations $\mathrm{h} \varepsilon \mathrm{G}$ and $\mathrm{g} \varepsilon \mathrm{G}$ such that $\mathrm{g}=\mathrm{f}^{*} \mathrm{~h}^{*} \mathrm{f}^{-}$ ${ }^{1}$ are said to be conjugate transformations. The conjugate set of transformations split the group $\mathrm{G}$ into disjoint sets. There is another way of partitioning a group into disjoint sets using cosets. A coset is a subgroup of the group $\mathrm{G}$ obtained by choosing a subgroup $\mathrm{H}$ of the group first and then choosing another element a $\epsilon \mathrm{G}$, operate ' $\mathrm{a}$ ' on $\mathrm{H}$ defined by the group G. If a is operated on the left(or right), then it is a left(or right) coset aH. This method is used by wavelets, with the mother wavelet the chosen subgroup of the transformation group G. Fourier transform is defined for cyclic transformation groups, where each element is commutative with every other element and also called the abelian group.

Fourier transform [22][25][26] is also extended to non-abelian groups, where each transform is reduced to the trace of its matrix representation due to which it lags the functionality to localize frequencies in time. If the conjugate partitioning of a group is used then no approximation is needed. An abstract group is brought alive by choosing any basis in the vector space of the signal. The group itself contains the linear transformations on this vector space. Conjugate transformations in the group collect together the corresponding invariant subspaces of the vector space by deriving the irreducible representations of each linear transformation. These irreducible representations form the new basis for the signal called the G-lets. G-lets are not a single transform, but a set of transformations related by the rules of Group theory.

\subsection{Constructing G-Lets}

First a transformation group has to be chosen for generating G-lets. We focus only on finite groups whose representations are unitary so that they are always completely reducible into irreducible representations. A finite discrete signal is considered. The signal exists in a vector space $V$, which shall henceforth be called as the signal space. For this signal, we generate a finite set of G-lets and project the signal on them. The signal can be reconstructed from G-lets without any loss as shown in Fig:1 below individually as well as through a linear sum of the transforms.

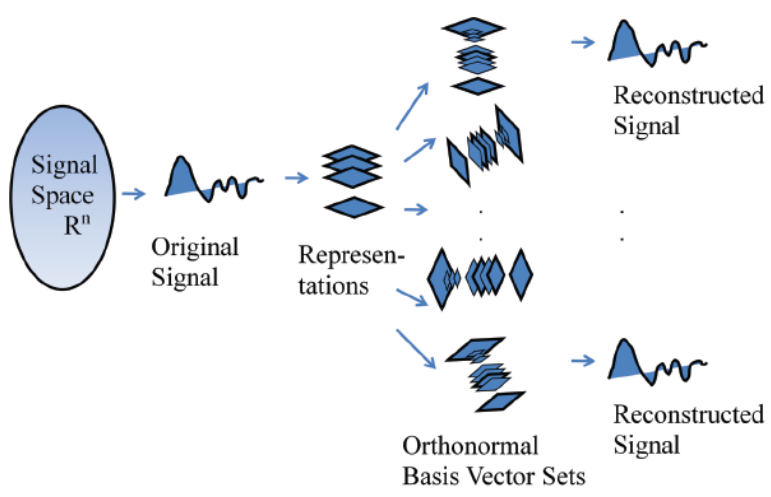

Fig 1: Representation matrices form a kaliedoscope
In the examples below the standard basis of the vector space $\mathrm{V}$ is the chosen basis of the signal given by

$$
\mathrm{L}=\sum_{\mathrm{i}=1}^{\mathrm{n}} \mathrm{e}_{\mathrm{i}} \quad \text { where } \mathrm{e}_{\mathrm{j}}=\{1 \text { at } \mathrm{i}=\mathrm{j}, 0 \text { otherwise }\}
$$

With this basis, the linear transformations materialize into matrices. The matrix representation looks like this:

$$
D(R)=\left[\begin{array}{llll}
D_{1}(R) & & & 0 \\
& D_{2}(R) & & \\
0 & & D_{3}(R) & \\
0 & & & \ldots
\end{array}\right]
$$

Each diagonal block $D_{i}(R)$ of the representation corresponds to one irreducible representation. The procedure to find the irreducible representations is given below.

By Schur's orthogonality lemma, the irreducible representations all put together are orthogonal and form a basis for the vector space $V$.

Schur's orthogonality lemma 1: For finite dimensional representation of a finite group $\mathrm{G}$ in which inner products have been introduced to make the representations unitary

- If R1 and R2 are two inequivalent irreducible representations, then every matrix coefficient of $R_{1}$ is orthogonal to every matrix coefficient of $R_{2}$ with respect to the inner product in the vector space $\mathrm{V}$.

- If $\mathrm{R}$ is another irreducible representations, then $\left(\frac{|G| \text { trace }(R)}{\operatorname{dim} V}\right)$ is equal to the action of $R$ on the other irreducible representations $\mathrm{R}_{1}, \mathrm{R}_{2}$.

As a consequence, the dimensions of the irreducible representations follow the rule [2] [5]:

$$
\sum_{i=1}^{m} d_{i}^{2}=D_{m}
$$

Where $d_{i}=$ dimension of $i^{\text {th }}$ irreducible representation, $D_{m}=$ dimension of representation matrix and $\mathrm{m}=$ number of irreducible representations.

To show an example, we consider 'Dihedral' groups which include only two transformations, namely rotations and reflections. The conjugate transformations of the Dihedral groups occur either in pairs or remain single. Therefore the dimension of an irreducible representation is either one or two. If the dimension of the signal is ' $n$ ', then the basis is also of the same dimension. The representation matrix of the linear transformation is chosen to be of size $\mathrm{n} \times \mathrm{n}$. The set of representation matrices for the transformations in the group form what is called the representation space of dimension $\mathrm{n}^{2}$. In a dihedral group $D_{n}$, there are ' $n$ ' rotations and ' $n$ ' reflections. Therefore there are $2 n$ matrices one for each transformation. The rotation matrix is given by

$$
\mathrm{R}_{\theta}=\left(\begin{array}{cc}
\cos \theta & -\sin \theta \\
\sin \theta & \cos \theta
\end{array}\right)
$$

The irreducible representations are lined up along the diagonal of a representation matrix. An example of the representation matrix where $n=9$ is given below and the rest of the matrices are given in the appendix. 
$\mathbf{R}_{1}=\left|\begin{array}{ccccccccc}0.766 & 0 & 0 & 0 & 0 & 0 & 0 & 0 \\ 0 & 0.766 & 0.6428 & 0 & 0 & 0 & 0 & 0 & 0 \\ 0 & 0.6428 & 0.766 & 0 & 0 & 0 & 0 & 0 & 0 \\ 0 & 0 & 0 & 0.766 & 0.6428 & 0 & 0 & 0 & 0 \\ 0 & 0 & 0 & 0.6428 & 0.766 & 0 & 0 & 0 & 0 \\ 0 & 0 & 0 & 0 & 0 & 0.766 & 0.6428 & 0 & 0 \\ 0 & 0 & 0 & 0 & 0 & 0.6428 & 0.766 & 0 & 0 \\ 0 & 0 & 0 & 0 & 0 & 0 & 0 & 0.766 & -0.6428 \\ 0 & 0 & 0 & 0 & 0 & 0 & 0 & 0.6428 & 0.766\end{array}\right|$

For dihedral groups, the number of irreducible representations is given by $(n+6) / 2$ for even sized ' $n$ ' and $(n+3) / 2$ for odd sized ' $n$ '. The matrix so formed is naturally a sparse representation. For other kinds of transformation groups, dimension of the corresponding irreducible representations are greater than two. Therefore the representation matrix can look like in the figure below with varying sizes of irreducible representations.

There are other transformation groups with combinations like rotations and translations, Heisenberg groups and etc. For illustration the G-lets for a 6-tuple sig $=\left\{\begin{array}{lllll}0.2 & 0.3 & 0.1 & 1.2 & 0.1\end{array}\right.$ 0.6 is shown in the Fig:2, Fig:3 and Fig:4.

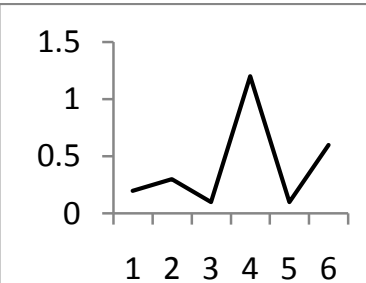

Fig 2: Original 6-tuple signal
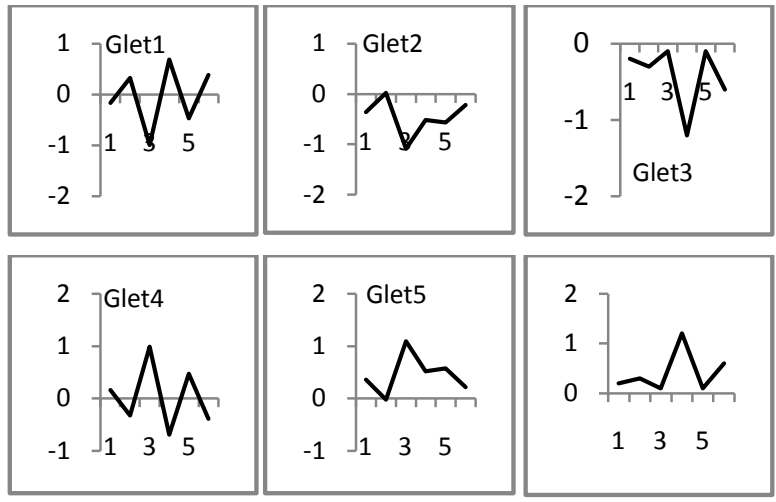

Fig 3: Rotation G-let coefficients
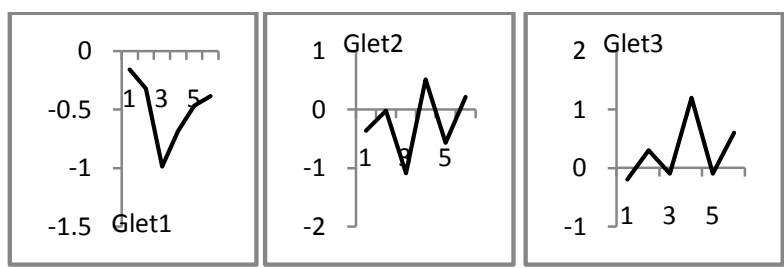
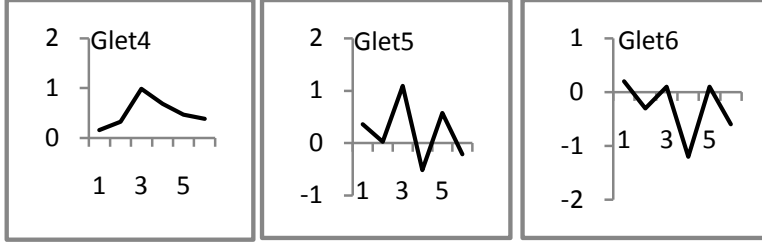

Fig 4: Reflection G-let coefficients

The figure above contains the original signal and both the Glets corresponding to rotations and reflections respectively. More examples, of a 3-tuple and 9-tuple signal, with their Glet coefficients and representation matrices are shown in the appendix below. A comparison of G-let coefficients for a 9tuple signal with Fourier and wavelet coefficients is shown in the following tables. Table 1 shows the G-let coefficients. Table 2 shows the Fourier and Wavelet coefficients for the same 9-tuple.

Table 1: Rotation G-let Coefficients

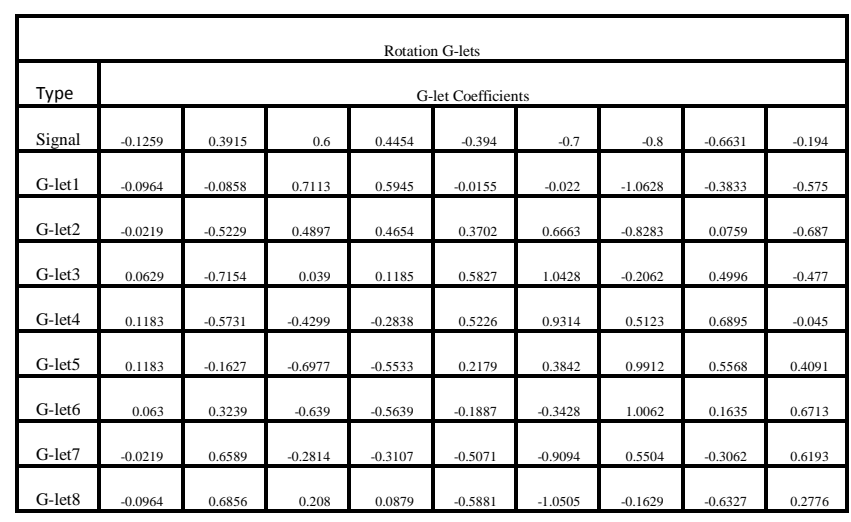

Table 2: Fourier and Wavelet Coefficients

\begin{tabular}{|l||lllll||}
\hline \multicolumn{1}{|l||}{ Type } & \multicolumn{5}{|c||}{ Coefficients } \\
\hline \hline Original & 0.1259 & 0.3915 & 0.6000 & 0.4454 & -0.3940 \\
Signal & -0.7000 & -0.8000 & -0.6631 & -0.1940 & \\
\hline \hline Fourier & -1.4401 & 1.2198 & $-2.8035 \mathrm{i}$ & $-0.6931+0.2666 \mathrm{i}$ \\
& $-0.0007+0.3218 \mathrm{i}$ & -0.3725 & $-0.1655 \mathrm{i}$ & -0.3725 \\
& $+0.1655 \mathrm{i}$ & -0.0007 & $-0.3218 \mathrm{i}$ & $-0.6931-0.2666 \mathrm{i}$ \\
& $1.2198+2.8035 \mathrm{i}$ & & & \\
\hline \hline Wavelet & 0.0049 & 0.3435 & 0.6646 & -0.8694 & -0.9594 \\
& -0.3011 & -0.3168 & 0.2153 & -0.1690 & -0.1411 \\
& 0.1836 & -0.0997 & & & \\
\hline
\end{tabular}

\section{SALIENT FEATURES}

Some of the salient features of this algorithm are discussed below:

- It may be seen in Figure that the signal is portrayed by representation matrices from different perspectives. The irreducible pieces might be big or small and that depends on the size of the signal. When a signal shows an irreducible representation of a large block size, it is possible to create an equivalent representation through a linear transformation with the help of its subspaces (null space or range space) such that the block becomes further reducible. This technique allows us to focus and explore further that part of the signal captured by the 
irreducible block as shown in Figures. The features of the signal under consideration are found to be spread across conjugacy classes, i.e., here they are spread across two conjugacy classes.
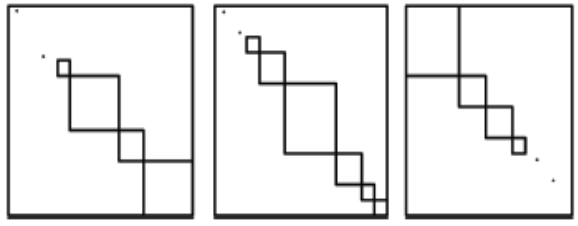

Fig 5: Irreducible blocks forming representation matrix

- The number of irreducible representations is equal to the number of conjugacy classes. Irreducible representations form the orthonormal basis. Conjugacy classes represent the structural symmetries for the signal. So, whenever a change occurs in the signal at a particular point, the above relationship propagates this change across different conjugacy classes.

- When a representation is chosen for a signal, the different basis of the underlying vector space gives different representations as if viewing through a kaleidoscope. We get a powerful method for matching the features of two signals especially in applications like pattern matching and face recognition with such a variety of basis sets.

- Expanding any function $f(x)$ in terms of the basis functions of irreducible representations requires that the representation group is obtained by applying to the function $f(x)$ all the transformations. Then the function is linearly expressible in terms of the basis functions in the various irreducible representations. One may also choose one of the irreducible representation functions $\mathrm{g}(\mathrm{x})$ with its projection of the signal and generate a new set of basis functions which will include itself in the set of basis functions. This feature allows us to customize the representation for any signal.

- As the size of the signal increases, the number of conjugacy classes is almost $50 \%$ less. This promises a compression of the signal, though further attention is needed in this regard. The Table 3 shows the number of conjugacy classes for different tuples.

Table 3: Number of Conjugacy Classes

\begin{tabular}{||l||l||l||l||}
\hline \hline S.No & $\begin{array}{l}\text { Tuple } \\
(\mathrm{n})\end{array}$ & Odd/Even & $\begin{array}{l}\text { No. of } \\
\text { conjugacy } \\
\text { classes (c) }\end{array}$ \\
\hline \hline 1 & 10 & Even & 8 \\
\hline \hline 2 & 21 & Odd & 12 \\
\hline \hline 3 & 32 & Even & 19 \\
\hline \hline 4 & 51 & Odd & 27 \\
\hline \hline 5 & 100 & Even & 53 \\
\hline \hline 6 & 501 & Odd & 252 \\
\hline \hline 7 & 1000 & Even & 503 \\
\hline
\end{tabular}

- In determining irreducible representations, a set of reducible representations may be divided into lower angle matrices and higher angle matrices. The lower angle matrices may then be explored using a completely different transformation group to further extract more features from that part of the signal.

- Group representation theory states that, new representations can be generated by direct product or tensor product of any two representation groups. Then their irreducible representations also turn out to be a direct product or tensor product.

- It is also possible to use complex representation matrices so long as a suitable transformation group is chosen. This quality could lead to the discovery of a new set of features in a signal.

- Local features: The local features of a signal can be identified by looking at the gradient map of each G-let. The same can be applied to G-lets at the next level where the G-let at the first level becomes the input to the second level. At each level, smaller and smaller local features are obtained.

- Complexity: The computational complexity depends on the number of viewpoints that need to be explored, the choice of transformation group and size of the signal. The matrices obtained in all of the representations are sparse orthonormal block-diagonal matrices and this adds an advantage for large signal calculations. Only if all the angles are to be explored, do we need to compute all the representation matrices and their corresponding G-let coefficients. One member of every conjugacy class put together shows a complete and different view of the signal. The same can be achieved by the alternate members of the conjugacy classes. In this way, the number of G-lets required for reconstruction of the signal can be cut down to the number of conjugacy classes.

\section{CONCLUSION}

In this paper, a different approach to signal processing with the choice of a group of transformations is proposed. The results are demonstrated using the dihedral groups with rotations and reflections. The results provide a method of multiresolution analysis without an approximation like a mother wavelet. G-lets for other transformation groups need to be explored with a unique combination and characteristics and their suitability to different applications. G-lets can also be extended to a continuous signal in the Hilbert Space.

\section{REFERENCES}

[1] Rajathilagam, B., Murali Rangarajan, and Soman, K.P., 2011 G-lets: Signal Processing Algorithm Using Transformation Groups, IEEE Trans. Imag. Proc., submitted

[2] Riley, K. F., Hobson, M. P., and Bence, S. J., 2002 Mathematical Methods for Physics and Engineering, Cambridge University Press.

[3] Knapp, A. W., 1996 Group Representations and Harmonic Analysis, Part II, Amer. Math. Soc., 43, 537549.

[4] Duzhin, S.V., and Chebotarevsky, B.D., 2004 Transformation Groups for Beginners, Student Mathematical Library, 25.

[5] Hamermesh, M., 1989 Group Theory and Its Application to Physical Problems, New York, Dover Publications.

[6] Mallat, S., 1998 A Wavelet Tour of Signal Processing, Academic Press. 
[7] Fokas, A.S., 1997 A unified transform method for solving linear and certain nonlinear PDEs, Proc. R. Soc. London A., 453, 1411-1443.

[8] Daubechies, I., 1988 Orthonormal bases of compactly supported wavelets, Comm. Pure Appl. Math., 41, 909996, October.

[9] Daubechies, I., 1992 Ten Lectures on Wavelets, Society for Industrial and Applied Mathematics.

[10] Candes, E.J., 1998 Ridgelets: theory and applications, $\mathrm{PhD}$ thesis, Stanford University.

[11] Candes, E.J., and Donoho, D.L., 1999 Ridgelets: the key to high dimensional intermittency?, Phil. Trans. R. Soc. London A, 357, 2495-2509.

[12] Matus, F., and Flusser, J., 1993 Image representations via a finite Radon transform, IEEE Transactions on Pattern Analysis and Machine Intelligence, 15, 9961006.

[13] Candes, E.J., and Donoho, D.L., 2000 Curvelets and curvilinear integrals, J. Approx. Theory, 113, 59-90.

[14] Do, M. N., and Vetterli, M., 2003 Contourlets, Beyond Wavelets, Stoeckler, J, Welland, G. V., Ed., Academic Press.

[15] Donoho, D.L., 1999 Wedgelets: nearly-minimax estimation of edges, Ann. Statist, 27, 859-897.

[16] Mallat, S., 2009 Geometrical grouplets, Appl. Comp. Harmonic Analysis, 26, 161-180.

[17] Lenz,R., 1990 Group Theoretical Methods in Image Processing, Springer-Verlag New York, Inc.

[18] Lenz,R., and Latorre Carmona, P., 2009 Octahedral transforms for 3-D image processing, IEEE Trans. Image Processing, 18, 2618-2628, August.

[19] Vale, R., and Waldron, S., 2008 Tight frames generated by finite nonabelian groups, Numerical Algorithms, 48, 11-27, July.

[20] Starck, J.L., Elad, M., and Donoho, D., 2005 Image decomposition via the combination of sparse representation and a variational approach, IEEE Trans. Image Processing, 14, 1570-1582.

[21] Aharon, M., Elad, M., and Bruckstein, A.M., 2006 The K-SVD: An algorithm for designing overcomplete dictionaries for sparse representation, IEEE Trans. Signal Processing, 54, 4311-4322, November.

[22] Viana, M., and Lakshminarayanan, V., 2010 Dihedral Fourier analysis, Lecture Notes in Statistics, Springer, New York, NY.

[23] Dresselhaus, M. S., 2008 Group Theory, Springer.

[24] Hamermesh, M., 1965 Group theory and its applications to physical problems, Addison Wesley Publishing Company, Inc.

[25] Assefa, D., Mansinha, L., Tiampo, K. F., Rasmussen, H., and Abdella, K., 2010 Local quaternion Fourier transform and color image texture analysis, Signal Processing, 90,1825-1835, June.

[26] Stankovic, R. S., Moraga, C., and Astola, J., 1999 Readings in Fourier Analysis on Finite Non-Abelian Groups, TICSP Series,Sharp5, September.

\section{Appendix}

G-lets for a 3-tuple signal using the group $\mathrm{D}_{3}$ is shown below:

\begin{tabular}{|l|r|r|r|}
\hline \multicolumn{4}{|c|}{ Rotation G-lets } \\
\hline Type & \multicolumn{3}{|c|}{ Coefficients } \\
\hline Signal & 44 & 55 & 34 \\
\hline G-let1 & -22 & -56.9449 & 30.6314 \\
\hline G-let2 & -22 & 1.9449 & -64.6314 \\
\hline
\end{tabular}

\begin{tabular}{|l|r|r|r|}
\hline \multicolumn{4}{|c|}{ Reflection G-lets } \\
\hline Type & \multicolumn{3}{|c|}{ Coefficients } \\
\hline Signal & 44 & 55 & 34 \\
\hline G-let1 & 22 & -56.9449 & -30.6314 \\
\hline G-let2 & 22 & 1.9449 & 64.6314 \\
\hline
\end{tabular}

$R 1=\left|\begin{array}{rrr}-0.5 & 0 & 0 \\ 0 & -0.5 & -0.866 \\ 0 & 0.866 & -0.5\end{array}\right|$

$R 2=\mid \begin{array}{rrr}-0.5 & 0 & 0 \\ 0 & 0.5 & 0.866 \\ 0 & -0.866 & -0.5\end{array}$

$R 3=\left|\begin{array}{lll}1 & 0 & 0 \\ 0 & 1 & 0 \\ 0 & 0 & 1\end{array}\right|$

$R 4=\left|\begin{array}{rrr}0.5 & 0 & 0 \\ 0 & -0.5 & -0.866 \\ 0 & -0.866 & 0.5\end{array}\right|$

$R 5=\left|\begin{array}{rrr}0.5 & 0 & 0 \\ 0 & -0.5 & 0.866 \\ 0 & 0.866 & 0.5\end{array}\right|$

$R 6=\left|\begin{array}{rrr}-1 & 0 & 0 \\ 0 & 1 & 0 \\ 0 & 0 & -1\end{array}\right|$

G-lets for a nine tuple signal using the group D9 is shown as in Fig. The signal is chosen as

$\operatorname{sig}=\left[\begin{array}{lllll}-0.1259 & 0.3915 & 0.6000 & 0.4454 & -0.3940\end{array}\right.$ $\left.\begin{array}{llll}-0.7000 & -0.8000 & -0.6631 & -0.1940\end{array}\right]$

Since the 9th and the 18th representation matrices are the identity transformations, they have been omitted here. Also the rotation g-lets are shown in the discussion of constructing g-lets above. 


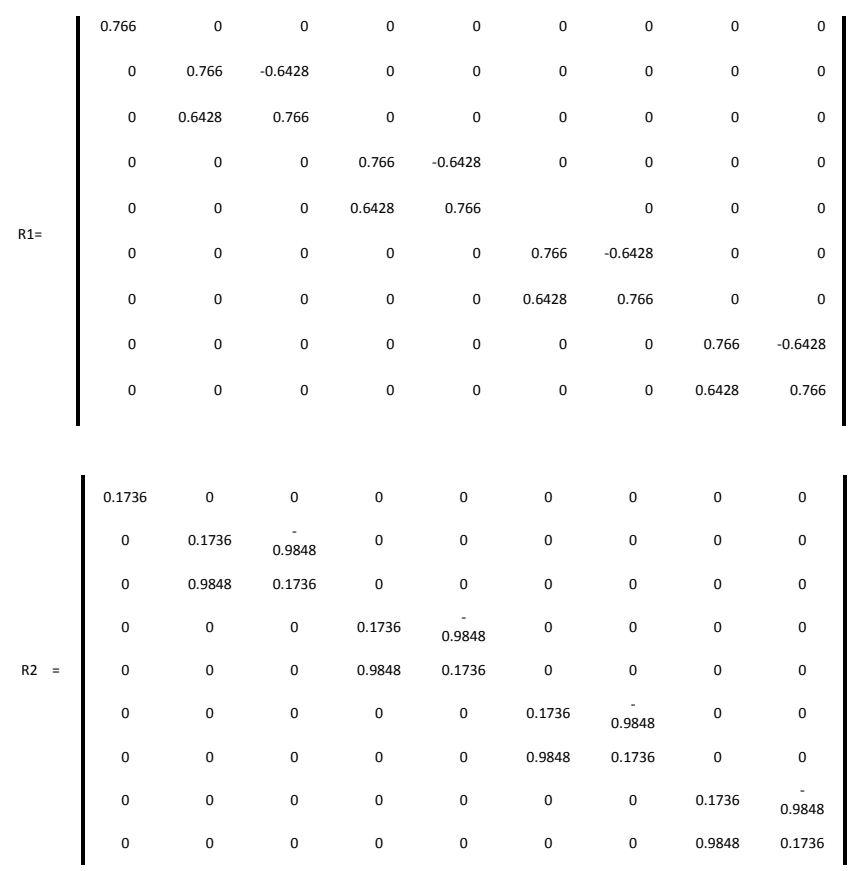

R6 $=\left(\begin{array}{rrrrrrrrr}-0.5 & 0 & 0 & 0 & 0 & 0 & 0 & 0 & 0 \\ 0 & -0.5 & 0.866 & 0 & 0 & 0 & 0 & 0 & 0 \\ 0 & -0.866 & -0.5 & 0 & 0 & 0 & 0 & 0 & 0 \\ 0 & 0 & 0 & -0.5 & 0.866 & 0 & 0 & 0 & 0 \\ 0 & 0 & 0 & -0.866 & -0.5 & 0 & 0 & 0 & 0 \\ 0 & 0 & 0 & 0 & 0 & -0.5 & 0.866 & 0 & 0 \\ 0 & 0 & 0 & 0 & 0 & -0.866 & -0.5 & 0 & 0 \\ 0 & 0 & 0 & 0 & 0 & 0 & 0 & -0.5 & 0.866 \\ 0 & 0 & 0 & 0 & 0 & 0 & 0 & -0.866 & -0.5\end{array} \mid\right.$

$\mathrm{R} 7=\left|\begin{array}{rrrrrrrrr}0.1736 & 0 & 0 & 0 & 0 & 0 & 0 & 0 & 0 \\ 0 & 0.1736 & 0.9848 & 0 & 0 & 0 & 0 & 0 & 0 \\ 0 & 0.9848 & 0.1736 & 0 & 0 & 0 & 0 & 0 & 0 \\ 0 & 0 & 0 & 0.1736 & 0.9848 & 0 & 0 & 0 & 0 \\ 0 & 0 & 0 & 0.9848 & 0.1736 & 0 & 0 & 0 & 0 \\ 0 & 0 & 0 & 0 & 0 & 0.1736 & 0.9848 & 0 & 0 \\ 0 & 0 & 0 & 0 & 0 & 0.9848 & 0.1736 & 0 & 0 \\ 0 & 0 & 0 & 0 & 0 & 0 & 0 & 0.1736 & 0.9848 \\ 0 & 0 & 0 & 0 & 0 & 0 & 0 & 0.9848 & 0.1736\end{array}\right|$

$R 3=\left|\begin{array}{rrrrrrrrr}-0.5 & 0 & 0 & 0 & 0 & 0 & 0 & 0 & 0 \\ 0 & -0.5 & -0.866 & 0 & 0 & 0 & 0 & 0 & 0 \\ 0 & 0.866 & -0.5 & 0 & 0 & 0 & 0 & 0 & 0 \\ 0 & 0 & 0 & -0.5 & -0.866 & 0 & 0 & 0 & 0 \\ 0 & 0 & 0 & 0.866 & -0.5 & 0 & 0 & 0 & 0 \\ 0 & 0 & 0 & 0 & 0 & -0.5 & -0.866 & 0 & 0 \\ 0 & 0 & 0 & 0 & 0 & 0.866 & -0.5 & 0 & 0 \\ 0 & 0 & 0 & 0 & 0 & 0 & 0 & -0.5 & -0.866 \\ 0 & 0 & 0 & 0 & 0 & 0 & 0 & 0.866 & -0.5\end{array}\right|$

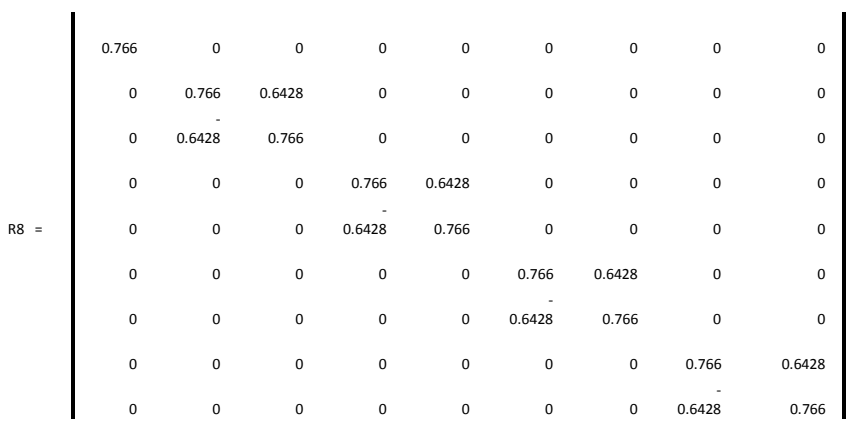

$R 4=\left(\begin{array}{rrrrrrrrr}0.9397 & 0 & 0 & 0 & 0 & 0 & 0 & 0 & 0 \\ 0 & 0.9397 & -0.342 & 0 & 0 & 0 & 0 & 0 & 0 \\ 0 & 0.342 & 0.9397 & 0 & 0 & 0 & 0 & 0 & 0 \\ 0 & 0 & 0 & 0.9397 & -0.342 & 0 & 0 & 0 & 0 \\ 0 & 0 & 0 & 0.342 & 0.9397 & 0 & 0 & 0 & 0 \\ 0 & 0 & 0 & 0 & 0 & 0.9397 & -0.342 & 0 & 0 \\ 0 & 0 & 0 & 0 & 0 & 0.342 & 0.9397 & 0 & 0 \\ 0 & 0 & 0 & 0 & 0 & 0 & 0 & 0.9397 & -0.342 \\ 0 & 0 & 0 & 0 & 0 & 0 & 0 & 0.342 & 0.9397\end{array} \mid\right.$

\begin{tabular}{|c|c|c|c|c|c|c|c|c|c|}
\hline \multicolumn{10}{|c|}{ Reflection G-lets } \\
\hline Type & \multicolumn{7}{|c|}{ Coefficients } \\
\hline Signal & -0.1259 & 0.3915 & 0.6 & 0.4454 & -0.394 & -0.7 & -0.8 & -0.6631 & -0.194 \\
\hline G-let1 & 0.0964 & -0.0858 & 0.7113 & 0.5945 & -0.0155 & 0.022 & -0.0628 & 0.3833 & 0.5748 \\
\hline G-let2 & 0.0219 & -0.5229 & -0.4897 & 0.4654 & -0.3702 & 0.6663 & 0.8283 & 0.0759 & 0.6867 \\
\hline G-let3 & -0.0629 & -0.7154 & -0.039 & 0.1185 & -0.5827 & 1.0428 & 0.2062 & 0.4996 & 0.4773 \\
\hline G-let4 & -0.1183 & -0.5731 & 0.4299 & -0.2838 & -0.5226 & 0.9314 & -0.5123 & 0.6895 & 0.0445 \\
\hline G-let5 & -0.1183 & -0.1627 & 0.6977 & -0.5533 & -0.2179 & 0.3842 & -0.9912 & 0.5568 & -0.4091 \\
\hline G-let6 & -0.063 & 0.3239 & 0.639 & -0.5639 & 0.1887 & -0.3428 & -1.0062 & 0.1635 & -0.6713 \\
\hline G-let7 & 0.0219 & 0.6589 & 0.2814 & -0.3107 & 0.5071 & -0.9094 & -0.5504 & -0.3062 & -0.6193 \\
\hline G-let8 & 0.0964 & 0.6856 & -0.208 & 0.0879 & 0.5881 & -1.0505 & 0.1629 & -0.6327 & -0.2776 \\
\hline
\end{tabular}

R5 $=\left(\begin{array}{rrrrrrrrr}0.9397 & 0 & 0 & 0 & 0 & 0 & 0 & 0 & 0 \\ 0 & 0.9397 & 0.342 & 0 & 0 & 0 & 0 & 0 & 0 \\ 0 & -0.342 & 0.9397 & 0 & 0 & 0 & 0 & 0 & 0 \\ 0 & 0 & 0 & 0.9397 & 0.342 & 0 & 0 & 0 & 0 \\ 0 & 0 & 0 & -0.342 & 0.9397 & 0 & 0 & 0 & 0 \\ 0 & 0 & 0 & 0 & 0 & 0.9397 & 0.342 & 0 & 0 \\ 0 & 0 & 0 & 0 & 0 & -0.342 & 0.9397 & 0 & 0 \\ 0 & 0 & 0 & 0 & 0 & 0 & 0 & 0.9397 & 0.342 \\ 0 & 0 & 0 & 0 & 0 & 0 & 0 & -0.342 & 0.9397\end{array} \mid\right.$ 
Another example for G-lets of an ECG signal is shown here The signal is a 168-tuple.

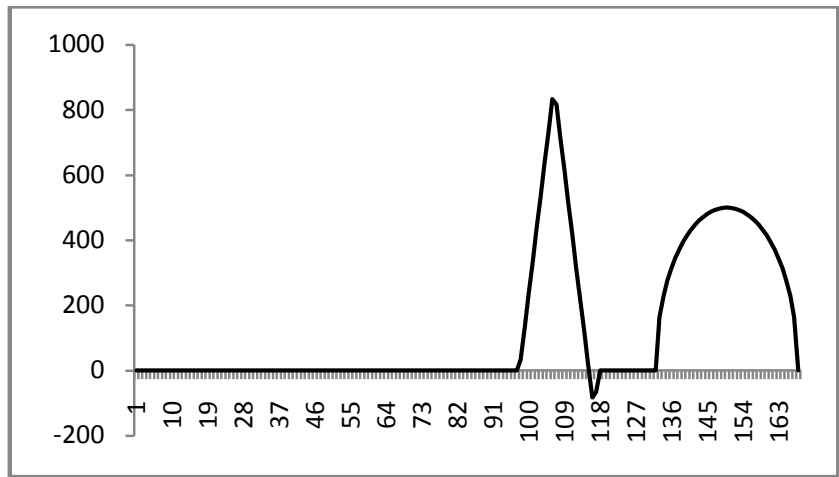

Some of the g-lets for this signal are shown below. The $1^{\text {st }} \mathrm{g}$ let is given by

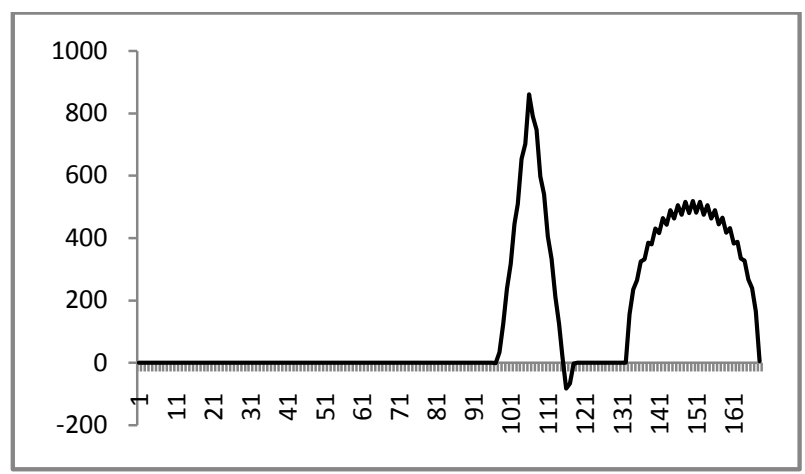

The $10^{\text {th }} \mathrm{g}$-let is given by

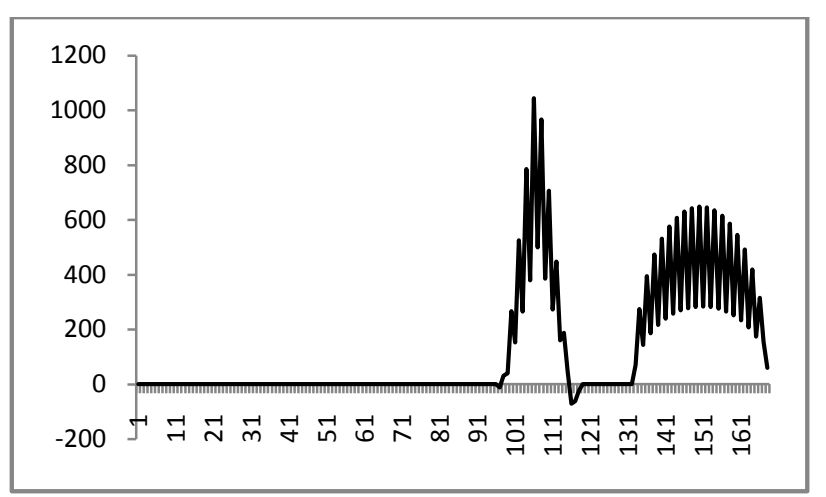

The $50^{\text {th }} \mathrm{g}$-let is given by

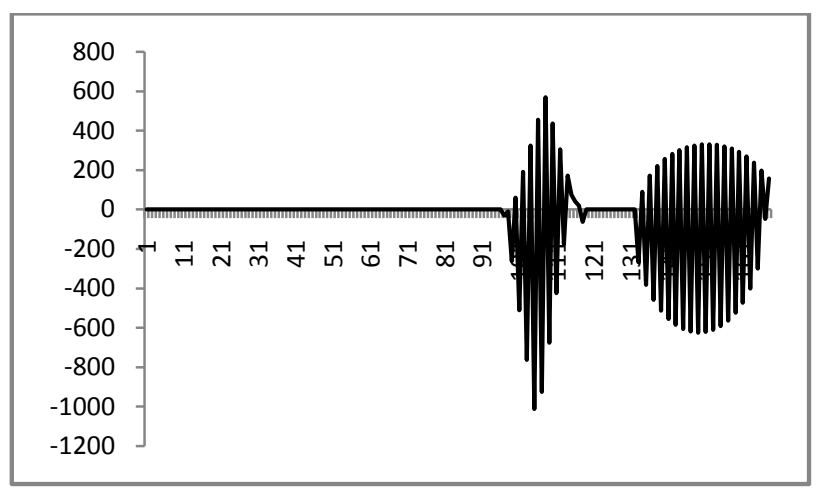

The $100^{\text {th }}$ g-let is given by

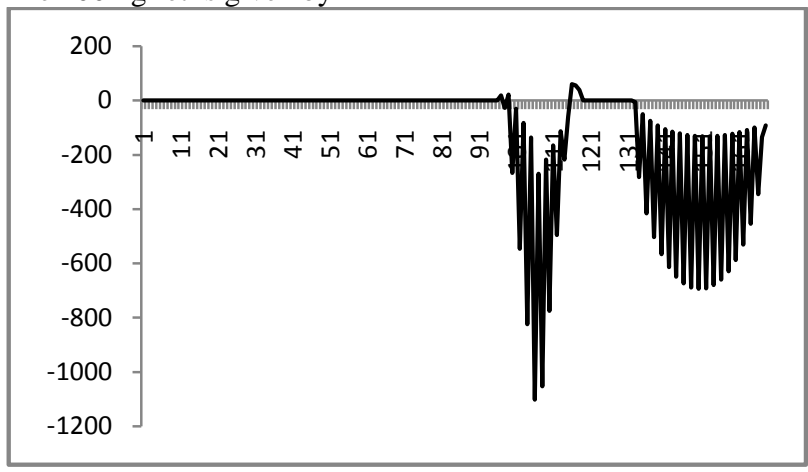

The above are rotation g-lets. The reflection g-lets are also shown here. The $170^{\text {th }} \mathrm{g}$-let is given by

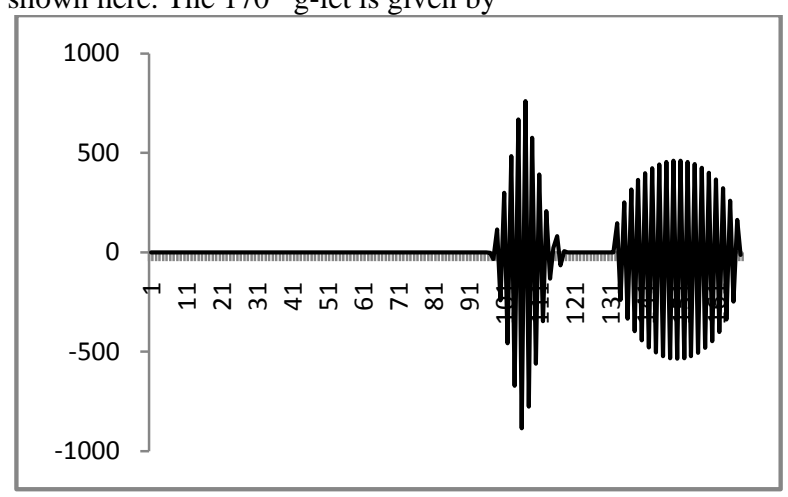

The $200^{\text {th }} \mathrm{g}$-let is given by

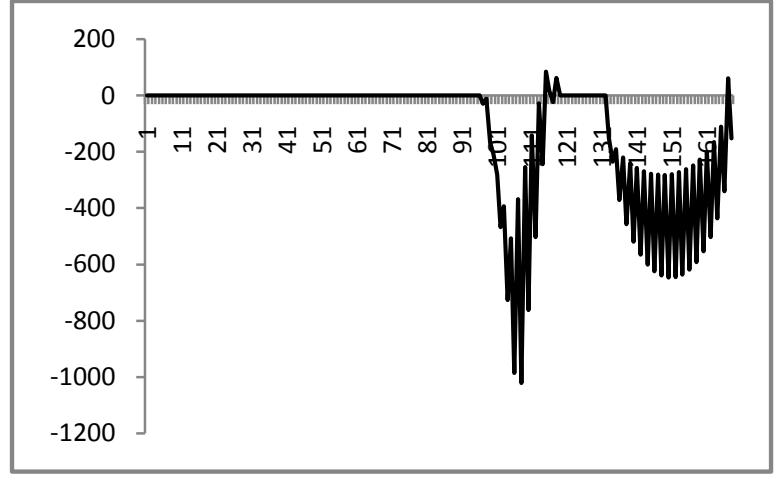

The $300^{\text {th }} \mathrm{g}$-let is given by

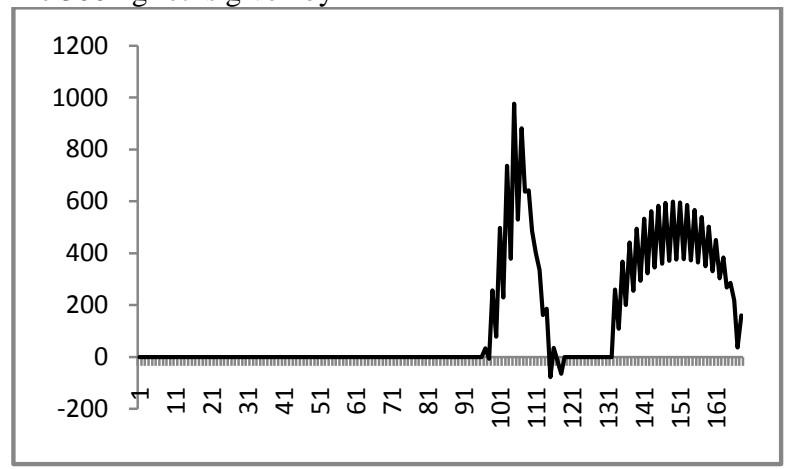

In the above figures we see that there are oscillations in the signal. This is due to the two dimensional irreducible representations. The oscillations originate at a specific point in the signal and spread across the signal. This feature helps us to identify low and high frequencies. Filters can also be designed using various transformation groups. 